\title{
Prediction Model for Health-Related Fitness Status Using Discriminant Analysis
}

\author{
Bayu Agung Pramono ${ }^{1, *}$ Yetty Septiani Mustar ${ }^{2,}$ Hijrin Fitroni $^{2,}$ Nurhasan $^{3}$ \\ ${ }^{1}$ Department of Sport Coaching Education, Universitas Negeri Surabaya \\ ${ }^{2}$ Department of Health Education and Recreation, Universitas Negeri Surabaya \\ ${ }^{3}$ Department of Sport Education, Universitas Negeri Surabaya \\ *Corresponding author. Email: bayupramono@unesa.ac.id
}

\begin{abstract}
Physical fitness represents the well-being of an individual and figures in measuring individual daily activities. This research aims to differentiate the physiological variables in distinguishing man fitness status without physical tests. The subjects of the examination amounted to 60 men aged 17-23 years old. The physiological parameters were age, weight, height, resting heart rate, blood glucose level, blood pressure, and VO2max, a bio-motoric component, measured by cooper protocol, running for $2.4 \mathrm{~km}$. Subsequently, seven physiological and bio-motoric segments were analyzed using discriminant analysis. The result showed that resting heart rate and weight are strongly correlated with individual fitness. Discriminant analysis applying a stepwise method demonstrated that resting heart rate and weight were included in the fitness component formula. Furthermore, discriminant analysis results prove that 59.57 (98.3\%) discriminant formulas can distinguish male fitness status. Fitness is an essential indicator in monitoring health. Concerning this, it is imperative to increase public awareness to regularly monitor their health status by assessing fitness status without having to do a physical test.
\end{abstract}

Keywords: Fitness status, Prediction variable, Discriminant analysis.

\section{INTRODUCTION}

Today knowing physical status is essential because it describes the body's immune ability to defend itself from virus attacks [1]. Regular physical exercise of fewer than 60 minutes is crucial in stimulating immune enhancement [2]. Lack of physical activity is the leading cause of death [3]. WHO reports that around 3.2 million people die each year from lack of physical activity [4]. Physical exercise is currently the best treatment for people to survive the virus attack.

Physical activity is a body movement that results from skeletal muscle in which energy use is required [5]. Participation in sports could positively contribute to human physiology, cognition, and psychology [6]. Regular physical activity can improve the quality of life by considering the time and the intensity [7]. Irregular exercise patterns may cause trouble in metabolism and, eventually, fitness. This disturbance occurs due to the lack of exercising strength, balance, endurance, agility, mobility, and flexibility training [8].

Fitness monitoring becomes a crucial step to performing and maintaining physical fitness. A fitness test typically uses a running test as an instrument, coupled with considering some rigorous methods. However, this test calls for tools, devices, and protocols that are both varied and advanced [9]. Coaches, talent scouts, government, and sports-oriented society demand a simple but accurate and flexible measurement method that can be performed in a situation with many samples simultaneously [10].

VO2max increment is a way to assess a fitness body that, in the end, could affect health. Aerobic capacity or VO2max has long been deemed a health predictor of detrimental illness, such as cardiovascular disease and other lethal diseases [11]. VO2max are important indicators too for the prevalence of carotid atherosclerosis [12].

Additionally, this research is important because home isolation from COVID19 is reported to have led to a reduction in all levels of physical activity, an increase in daily sitting time by approximately $28 \%$, and an increase in consumption patterns. unhealthy food [13]-[15]. Therefore, we need to monitor health indirectly at all times. Controlling body size and body shape by doing extra exercise benefits children in physical fitness and motor performance [16]. Monitoring, especially physical fitness monitoring, will become a common feature of 
many physical education courses [17]. Physical health monitoring is an important task that must be carried out continuously [18].

Current fitness tests using the physical examination are conducted using direct and indirect methods and apply an indicator of the limit of individual capability in maximizing oxygen for metabolism energy or widely recognized as VO2max. This test requires individuals to exercise physical activity beforehand for acquiring their fitness status.

\section{METHODS}

The subjects were 60 active men that in a week only perform two until three times physical exercises. The resting heart rate was counted using cardiac telemetry, weight was scaled using a bodyweight scale, height was measured using a stature meter, and blood pressures were measured using a mercury sphygmomanometer. The fitness test was carried out by following a copper test running for 2,4 Km. Data analysis in this research used SPSS 26, and normality tests were carried out beforehand to comprehend data distribution using one sample KS (Kolmogorov-Smirnov). Following this, discriminant analysis is used to obtain a fitness data predictor. Test of significance was conducted on discriminant analysis by using a chi-square test to identify fitness status. Linear Discriminant Analysis (LDA) is a simple and effective pattern classification technique, and it is also widely used for early disease detection using electronic medical record (EHR) data [19]. Written informed consent has been obtained. People with a history of heart disease, lung disease, smokers, and medications for chronic diseases were excluded from the study. All procedures followed are in line with the Helsinki Declaration as revised in 2013 [20].

\section{RESULTS}

Table 1. The characteristic of the subject

\begin{tabular}{|c|c|c|}
\hline \multirow{2}{*}{ Characteristic of subject } & \multicolumn{2}{|c|}{$n=60$} \\
\hline & Min - Max & Mean \pm SD \\
\hline Age (year) & $17.00-23.00$ & $19.78 \pm 1.35$ \\
\hline Weight (kg) & $44.70-70.00$ & $58.70 \pm 5.22$ \\
\hline Height (cm) & $159.00-180.00$ & $166.95 \pm 4.76$ \\
\hline Resting Heart Rate (beat/minute) & $51.00-91.00$ & $64.83 \pm 7.93$ \\
\hline Glucose Blood Rate (mg/dl) & $64.00-100.00$ & $86.73 \pm 8.98$ \\
\hline Systole $(\mathrm{mmHg})$ & $100.00-125.00$ & $115.92 \pm 5.41$ \\
\hline Diastole (mmHg) & $60.00-80.00$ & $69.50 \pm 5.02$ \\
\hline
\end{tabular}

Note. $\mathrm{n}$ - sample size, SD - Standard Deviation

Table 1 reflects the average variable discriminator of the research subject characteristic, namely age $19.78 \pm 1.35$ years, weight $58.70 \pm 5.22 \mathrm{~kg}$, height
$166.95 \pm 4.76 \mathrm{~cm}$, resting health rate $64.83 \pm 7.93$ beats per minute, blood glucose level $86.73 \pm 8.98 \mathrm{mg} / \mathrm{dl}$, systole $115.92 \pm 5.41 \mathrm{mmHg}$, and diastole $69.50 \pm 5.02 \mathrm{mmHg}$.

Table 2. Normality test

\begin{tabular}{|c|c|c|c|c|c|c|c|}
\hline & Age & Wg & Hg & RH & GB & Sys & Ds \\
\hline KS & 1.17 & 0.66 & 1.23 & 1.30 & 0.99 & 2.90 & 1.17 \\
\hline Sig. & 0.12 & 0.77 & 0.09 & 0.06 & 0.27 & 0.00 & 0.12 \\
\hline
\end{tabular}

Note. Weight - Wg, Height - Hg, Resting Heart rate - RH, Glucose Blood - GB, Systole - Sys, Diastole-Ds.

The normality test in table 2 illustrates that only age, weight, height, resting heart rate, and glucose blood rate was typically distributed because of $\mathrm{p}>0.05$.
Consequently, these five data could be used in multivariate correlation and discriminant analysis. 
Table 3. Statistical results of the correlation test

\begin{tabular}{|l|c|c|}
\hline & \multicolumn{2}{|c|}{$V_{2} \max$} \\
\hline Age & $r$ & $p$ \\
\hline Resting heart rate & -0.50 & $0.00^{*}$ \\
\hline Height & -0.72 & $0.00^{*}$ \\
\hline Weight & 0.04 & 0.37 \\
\hline Glucose blood rate & 0.25 & $0.02^{*}$ \\
\hline
\end{tabular}

Note. $\mathrm{r}$ - correlation coefficient, $p-p_{\text {value, }}$, indicates $\mathrm{p}<0.05$

The subsequent data analysis was followed by a correlation test among free and bound variables. Table 3 demonstrates that age, resting heart age, and weight was significantly correlated with VO2max. Those three variables affected the value of the VO2max sample. Considering the solid and inverse correlation, the less the importance of those three obtained, the more the volume of VO2max reached.

Table 4. Coefficient of discriminant function

\begin{tabular}{|l|c|}
\hline & Coefficient of function \\
\hline Resting heart rate & 0.205 \\
\hline Weight & -0.070 \\
\hline Constant & -9.182 \\
\hline
\end{tabular}

The statistic measurement displayed in table 4 shows the coefficient that could be included in the discriminant function model 1 .

$\mathrm{D}=-9.182+(0.205 \mathrm{x}$ resting heart rate $)+(-0.070 \mathrm{x}$ weight)
The discriminant model 1 will be used for gaining a discriminant score that functions in predicting an object classification into a fitness group in which a score that is less than 0.0000167 includes a fit group. In contrast, a group with a value of more than 0.00 is an unfit group.

Table 5. Statistical results of significance test

\begin{tabular}{|c|c|c|c|}
\hline Wilks' Lambda & Chi-square & df & Sig. \\
\hline 0.352 & 59.575 & 2 & $0.000^{*}$ \\
\hline
\end{tabular}

Note. $\mathrm{df}$ - degree of freedom, sig. - significant, *indicates $\mathrm{p}<0.05$.

The level of significance estimated based on chisquare and transformed statistically in Table 5 shows that Wilks' Lambda was associated with discriminant function at 0.35 . This number then was converted to be a chi-square with a degree of freedom was amounted to 2 .
The chi-square value was 59.575. In light of that, it is reasonable to refute $\mathrm{H} 0$ with an error rate $\mathrm{p}=0.00$ and assert that the discriminant function succeeded in discriminating fitness status.

Table 6. Classification Results

\begin{tabular}{|l|c|c|c|c|c|}
\hline \multicolumn{2}{|c|}{} & \multirow{2}{*}{ code } & \multicolumn{2}{|c|}{ Predicted Group Membership } & \multirow{2}{*}{$n=60$} \\
\cline { 3 - 6 } & & fit & unfit & 29 \\
\hline \multirow{3}{*}{ Original } & \multirow{2}{*}{ Count } & Fit & 29 & 0 & 31 \\
\cline { 3 - 6 } & \multirow{2}{*}{$\%$} & Unfit & 1 & 30 & 100.0 \\
\cline { 2 - 6 } & & Fit & 100.0 & .0 & 100.0 \\
\cline { 2 - 6 } & & Unfit & 3.2 & 96.8 & \\
\hline
\end{tabular}

Note. $\mathrm{n}$ - sample size

Table 6. Demonstrates that discriminant function succeeded in differentiating fitness status with the accuracy in grouping individuals was $98.3 \%$. This table explains that from 60 samples following the physical test, 29 models were considered fit, and it was proven $100 \%$ using the discriminant formula, which also showed the 
fitness status. On the other hand, from 31 samples that were categorized in the unfit group, only $96.8 \%$ can be predicted, or only 30 models classified unfit based on the physical test. Therefore, one sample was predicted wrong by the discriminant formula.

\section{DISCUSSION}

Physical activities, notably aerobic activities, are the most effective way for non-communicable diseases. Physical activity could increase HDL production in mature humans that have lower HDL. An increase in HDL is helpful for non-communicable disease patients, such as heart attack [21], [22].

Increased physical activity will affect one's fitness. An increase in fitness is significantly effective in helping someone to maximize their routines. Individuals are highly recommended to know their fitness status to prepare their daily activities well. Typically, to measure it, physical tests, including running, are required. This test calls for several tools, methods, and specific protocols. Still, until now, an accurate but straightforward fitness assessment method has not existed yet, particularly for the activity affected by weight [23].

One of the roles of exercise regulation is weight loss programs. Improving asthma control, quality of life, and psychosocial symptoms help to clarify potential mechanisms for improved lung function, airways, and inflammation systemic in obese patients with asthma [24].

The research found that two-body characteristics, resting heart rate, and weight, affect one's fitness. The health of adolescents could be seen by resting heart rate since this method is way more straightforward than physical tests [25]. Increased cardiopulmonary fitness can reduce arterial stiffness mainly through resting heart rate. High muscle strength may have a detrimental effect on arterial stiffness, partially offset by a lower resting heart rate [26].

Elevated resting heart rate is an independent cardiovascular risk factor [26]-[28] positively associated with arterial stiffness [29], [30]. High RHR may be due to overactive sympathetic nerves, which can directly increase arterial stiffness by increasing the cyclic mechanical shear stress on the arterial wall [31]. Regular exercise to improve CRF can lower RHR, which may be a way to reduce arterial stiffness [26], [32], [33]. In addition, when adaptation is positive to training, the indices of heart rate and post-exercise variability associated with the vagus nerve, post-exercise heart rate recovery, and heart rate acceleration are significantly increased, thereby improving performance [34].
These HRV analyses can provide researchers with direct information about the contribution of the parasympathetic nerve (and, by extension, inferred knowledge about the assistance of the sympathetic nerve) on the impact of rest and post-exercise HR modulation [34].

Resting heart rate has a significant and robust correlation with $\mathrm{VO} 2 \max$ [35]. Aerobic training will provide a reasonably practical advantage on the decline of resting heart rate [36]. Moreover, another research showed that resting heart rate is the most reliable predictor of VO2max and the best way to determine cardiovascular fitness [37]. The non-exercise variables of age, body mass, and resting heart rate may significantly predict the endurance abilities of athletes (VO2 max) [38].

The correlation test shows that weight has a lower correlation and is significant with $\mathrm{VO} 2 \mathrm{max}$. It is in line with research that stated that there was a negative correlation between $\mathrm{BMI}$ and $\mathrm{VO} 2$ max and the increase in body fat due to the decline of $\mathrm{VO} 2 \mathrm{max}$ in younger people [25], [26]. An increasing BMI affects physical fitness and causes a decrease in VO2 max [41]. These results are supported by research that shows that overweight girls can significantly reduce physical selfefficacy and underperform [42]. Cardiorespiratory fitness (CRF) provides an advantage in mediating the risk of heart failure (HF) associated with BMI [43].

Any physical activity that improves cardiorespiratory health should enhance lean body mass and reduce fat mass [42] so that the ideal body weight will support increased fitness. This research shows that weight and resting heart rate were the most reliable predictor in predicting fitness. With the discriminant formula obtained, the equation of this statistical calculation could predict an individual accurately with a fit field test. 98.3\% was justified fit through fitness formula calculation based on discriminant statistical results.

\section{CONCLUSION}

The measurement of fitness that has been carried out through physical tests is quite heavy. This test makes many people reluctant to assess his fitness. As a result, their fitness status is not monitored, and it can affect their health status. This study has shown a formula that can predict an individual's fitness status. Discriminant analysis can help in classifying fitness categories in ordinary men without a physical test. The results of this study provide positive information between resting pulse rate and fitness status, which are both products of regular and programmed physical activity. The indicators are resting heart rate and body weight. Researchers suggest 
that future studies include other fitness variables and involve more samples, not exclusively men.

\section{AUTHORS' CONTRIBUTIONS}

BAP: study conception, design, data collection, and statistical analysis; YSM: statistical analysis, data interpretation and drafting of the manuscript; HF: study conception and data collection; N: study conception and design. All authors have read and approved the final manuscript.

\section{ACKNOWLEDGMENTS}

This research was supported by Kemenristekdikti Indonesia and Universitas Negeri Surabaya.

\section{REFERENCES}

[1] I. Ahmed, "COVID-19 - do exercise prescription, and maximal oxygen uptake (VO2 max) have a role in risk-stratifying patients?," Clin. Med. J. R. Coll. Physicians London, vol. 20, no. 3, pp. 282-284, 2020, DOI: 10.7861/clinmed.2020-0111.

[2] D. C. Nieman and L. M. Wentz, "The compelling link between physical activity and the body's defense system," J. Sport Heal. Sci., vol. 8, no. 3, pp. 201-

217, 2019, DOI: 10.1016/j.jshs.2018.09.009.

[3] D. Taylor, "Physical activity is medicine for older adults," Postgrad. Med. J., vol. 90, no. 1059, pp. 26-32, 2014, DOI: 10.1136/postgrad med-2012131366 .

[4] W. World Health Organization, "Diet and Physical Activity Factsheet. Secondary Diet and Physical Activity Factsheet," 2013. http://www.who.int/dietphysicalactivity/factsheet_i nactivity/en/index.html.

[5] R. Delgado-Gonzalo et al., "Physical activity," Seamless Healthcare Monitoring: Advancements in Wearable, Attachable, and Invisible Devices, $2017 \ldots$

[6] K. L. Smith, K. Carr, A. Wiseman, K. Calhoun, N. H. Mcnevin, and P. L. Weir, "Barriers Are Not the Limiting Factor to Participation in Physical Activity in Canadian Seniors," J. Aging Res., vol. 2012, p. 8 pages, 2012, DOI: 10.1155/2012/890679.

[7] Y. S. Mustar and I. H. Susanto, "Oxidative Stress: Comparing Sub-Maximal Physical Activities as a Self-Reminder for Human Health System," IOP Conf. Ser. Mater. Sci. Engineering, vol. 180, p. 012265, 2017, DOI: $10.1088 / 1742-$ 6596/755/1/011001.

[8] W. W. Huang, Ã. S. Perera, and J. Vanswearingen,
"Performance Measures Predict Onset of Activity of Daily Living," J. Am. Great. Soc., vol. 58, no. 5, pp. $\quad 844-852, \quad 2010, \quad$ DOI: $10.1111 / \mathrm{j} .1532-$ 5415.2010.02820.x.

[9] L. Dalleck and A. Dalleck, "Journal of Exercise Physiology online," J. Exerc. Physiol. online, vol. 11, no. 4, pp. 1-11, 2008.

[10] U. Dimkpa, "Post-exercise heart rate recovery: An index of cardiovascular fitness," J. Exerc. Physiol., vol. 12 , no. 1, pp. 10-22, 2009.

[11] S. J. Keteyian et al., "Peak aerobic capacity predicts prognosis in patients with coronary heart disease," Am. Heart J., vol. 156, no. 2, p. :292-300, 2008, DOI: 10.1016/j.ahj.2008.03.017.

[12] S. J. Kang and K. J. Ko, "Association between resting heart rate, VO 2 max and carotid intimamedia thickness in middle-aged men," IJC Hear. Vasc., vol. 23, p. 100347, 2019, DOI: 10.1016/j.ijcha.2019.100347.

[13] A. Ammar et al., "Effects of COVID-19 Home Confinement on Eating Behaviour and Physical Activity: Results of the" Nutrients, vol. 12, no. 1583, p. 13, 2020.

[14] L. de Oliveira Neto, H. M. Elsangedy, V. D. de Oliveira Tavares, C. V. S. Teixeira, D. G. Behm, and M. E. da Silva-Grigoletto, "\#TrainingInHome training at home during the COVID-19 (SARS$\mathrm{CoV}-2$ ) pandemic physical exercise and behaviorbased approach," Brazilian J. Exerc. Physiol., vol. 19, no. 2, pp. 9-19, 2020, [Online]. Available: http://faculty.css.edu/tboone2/asep/Russell.pdf.

[15] A. Ammar et al., "Effects of home confinement on mental health and lifestyle behaviors during the COVID-19 outbreak: Insights from the ECLBCOVID19 multicentre study," Biol. Sport, vol. 38, no. 1, pp. 9-21, 2021, DOI: 10.5114/biolsport.2020.96857.

[16] M. Giuriato, A. Kawczynski, D. Mroczek, N. Lovecchio, and A. Nevill, "Allometric association between physical fitness test results, body size/shape, biological maturity, and time spent playing sports in adolescents," PLoS One, vol. 16, no. 4 April, pp. 1-13, 2021, DOI: 10.1371/journal.pone.0249626.

[17] L. Cale, J. Harris, and M. H. Chen, "Monitoring health, activity, and fitness in physical education: its current and future state of health," Sport. Educ. Soc., vol. 19, no. 4, pp. 376-397, 2014, DOI: 10.1080/13573322.2012.681298.

[18] Z. Kekeeva, V. Burlykov, and S. Proshkin, "Physical fitness monitoring as an enhancing means of specialist`s training quality in higher education institution," Int. J. Environ. Sci. Educ., vol. 11, no. 16, pp. 9092-9100, 2016. 
[19] S. Yang et al., "Early detection of disease using electronic health records and fisher's Wishart discriminant analysis," Procedia Comput. Sci., vol. 140, pp. 393-402, 2018, DOI: 10.1016/j.procs.2018.10.299.

[20] World Medical Association, "Declaration of Helsinki, Ethical Principles for Scientific Requirements and Research Protocols," Bull. World Health Organ., vol. 79, no. 4, p. 373, 2013, [Online]. Available: https://www.wma.net/policiespost/wma-declaration-of-helsinki-ethicalprinciples-for-medical-research-involving-humansubjects/.

[21] M. Béland, K. L. Lavoie, S. Briand, U. J. White, C. Gemme, and S. L. Bacon, "Aerobic exercise alleviates depressive symptoms in patients with a major non-communicable chronic disease: a systematic review and meta-analysis," pp. 1-9, 2019, DOI: 10.1136/by sports-2018-099360.

[22] C. Couillard et al., "Effects of Endurance Exercise Training on Plasma HDL Cholesterol Levels Depend on Levels of Triglycerides," pp. 12261233, 2001.

[23] W. D. McArdle, F. I. Katch, and V. L. Katch, Exercise Physiology: Energy, nutrition and human performance, vol. 78, no. 2. 2010.

[24] P. D. Freitas et al., "The effects of exercise training in a weight loss lifestyle intervention on asthma control, quality of life and psychosocial symptoms in adult obese asthmatics: Protocol of a randomized controlled trial," BMC Pulm. Med., vol. 15, no. 1, pp. 1-11, 2015, DOI: 10.1186/s12890-015-0111-2.

[25] D. A. S. Silva, T. R. De Lima, and M. S. Tremblay, "Association between resting heart rate and healthrelated physical fitness in Brazilian adolescents," Biomed Res. Int., vol. 2018, pp. 1-10, 2018, DOI: $10.1155 / 2018 / 3812197$.

[26] H. L. Quan et al., "Resting heart rate and the association of physical fitness with carotid artery stiffness," Am. J. Hypertens., vol. 27, no. 1, pp. 6571, 2014, DOI: 10.1093/ajh/hpt161.

[27] P. Palatini et al., "Management of the hypertensive patient with elevated heart rate: Statement of the Second Consensus Conference endorsed by the European Society of Hypertension," J. Hypertens., vol. 34, no. 5, pp. 813-821, 2016, DOI: 10.1097/HJH.0000000000000865.

[28] P. Palatini et al., "Identification and management of the hypertensive patient with elevated heart rate: Statement of a European Society of Hypertension Consensus Meeting," J. Hypertens., vol. 24, no. 4, pp. 603-610, 2006, DOI: 10.1097/01.hjh.0000217838.49842.1e.

[29] H. Tomiyama et al., "Synergistic relationship between changes in the pulse wave velocity and changes in the heart rate in middle-aged Japanese adults: A prospective study," J. Hypertens., vol. 28, no. 4, pp. 687-694, 2010, DOI: 10.1097/HJH.0b013e3283369fe8.

[30] R. S. Cunha et al., "Association between high heart rate and high arterial rigidity in normotensive and hypertensive subjects," J. Hypertens., vol. 15, no. 12, pp. 1423-1430, 1997, DOI: 10.1097/00004872199715120-00009.

[31] Y. S. Chatzizisis, A. U. Coskun, M. Jonas, E. R. Edelman, C. L. Feldman, and P. H. Stone, "Role of Endothelial Shear Stress in the Natural History of Coronary Atherosclerosis and Vascular Remodeling. Molecular, Cellular, and Vascular Behavior," J. Am. Coll. Cardiol., vol. 49, no. 25, pp. 2379-2393, 2007, DOI: 10.1016/j.jacc.2007.02.059.

[32] G. Huang, X. Shi, J. A. Davis-Brezette, and W. H. Osness, "Resting heart rate changes after endurance training in older adults: A meta-analysis," Med. Sci. Sports Exerc., vol. 37, no. 8, pp. 1381-1386, 2005, DOI: $10.1249 / 01 . m s s .0000174899 .35392 .0 \mathrm{c}$.

[33] G. Huang, X. Shi, C. A. Gibson, S. C. Huang, N. A. Coudret, and M. C. Ehlman, "Controlled aerobic exercise training reduces resting blood pressure in sedentary older adults," Blood Press., vol. 22, no. 6, pp. 386-394, 2013, DOI: 10.3109/08037051.2013.778003.

[34] C. R. Bellenger, J. T. Fuller, R. L. Thomson, K. Davison, E. Y. Robertson, and J. D. Buckley, "Monitoring Athletic Training Status Through Autonomic Heart Rate Regulation: A Systematic Review and Meta-Analysis," Sport. Med., vol. 46, no. 10 , pp. 1461-1486, 2016, DOI: 10.1007/s40279-016-0484-2.

[35] E. Habibi, H. Dehghan, M. Moghiseh, and A. Hasanzadeh, "Study of the relationship between the aerobic capacity (VO2 $\max$ ) and the rating of perceived exertion based on the measurement of heartbeat in the metal industries Esfahan.," J. Educ. Health Promot., vol. 3, no. 1, pp. 55-55, 2014, DOI: 10.4103/2277-9531.134751.

[36] K. Kang, Seol-Jung; Kim, Eon-ho; Ko, "Effects of aerobic exercise on the resting heart rate, physical fitness, and arterial stiffness of female patients with metabolic syndrome," J. Phys. Ther. Sci., vol. 28, pp. 1764-1768, 2016

[37] J. Nauman, S. T. Aspenes, T. Ivar, L. Nilsen, and L. J. Vatten, "A Prospective Population Study of Resting Heart Rate and Peak Oxygen Uptake ( the HUNT Study, Norway )," J. pone, vol. 7, no. 9, pp. 1-9, 2019.

[38] A. Rexhepi, "Prediction of vo2max based on age, body mass, and resting heart rate Prediction of VO 2 max based on age, body mass, and resting heart 
rate," Hum. Move., vol. 15, no. 1, pp. 56-59, 2017, DOI: 10.2478/humo-2014-0003.

[39] H. Mondal and S. P. Mishra, "Effect of BMI, Body Fat Percentage and Fat-Free Mass on Maximal Oxygen Consumption in Healthy Young Adults," pp. 17-20, 2017, DOI:

[41] S. B. Kalyanshetti and S. Veluru, "A cross-sectional study of the association of body mass index and VO2 max by nonexercise test in medical students," Natl. J. Physiol. Pharm. Pharmacol., vol. 7, no. 2, pp. 228-231, 2017, DOI: 10.5455/njppp.2017.7.0825804092016.

[42] K. Umamaheswari, Y. Dhanalakshmi, S. Karthik, D. Niraimathi, S. V Umadevi, and N. A. John, "VO2 Max and Body Mass in Overweight and
10.7860/JCDR/2017/25465.10039.

[40] S. Radovanović, S. Kocić, G. Gajović, S. Radević, and M. Milosavljević, "The impact of body weight on aerobic capacity," vol. 11, no. November 2013, pp. 204-209, 2014.

Obese Young Adults,” Int. J. Physiol., vol. 5, no. 2, pp. 23-27, 2017.

[43] A. Pandey et al., "Body Mass Index and Cardiorespiratory Fitness in Mid-Life and Risk of Heart Failure Hospitalization in Older Age: Findings From the Cooper Center Longitudinal Study," JACC Hear. Fail., vol. 5, no. 5, pp. 367374, 2017, DOI: 10.1016/j.jchf.2016.12.021. 